\title{
COHOMOLOGY OF SHEAVES OF HOLOMORPHIC FUNCTIONS SATISFYING BOUNDARY CONDITIONS ON PRODUCT DOMAINS $\left({ }^{1}\right)$
}

BY

\section{ALEXANDER NAGEL}

ABSTRACT. This paper considers sheaves of germs of holomorphic functions which satisfy certain boundary conditions on product domains in $\mathrm{C}^{n}$. Very general axioms for boundary behavior are given. This includes as special cases $L^{p}$ boundary behavior, $1<p<\infty$; continuous boundary behavior; differentiable boundary behavior of order $m, 0<m<\infty$, with an additional Hölder condition of order $a, 0 \leq \alpha \leq 1$, on the $m$ th derivatives. A fine resolution is constructed for those sheaves considered, and the main result of the paper is that all higher cohomology groups for these sheaves are zero.

Let $W_{i} \subset \subset \mathrm{C}$ be relatively compact domains for $i=1,2, \ldots, n$, and let $W=W_{1} \times W_{2} \times \cdots \times W_{n} \subset \subset \mathbf{C}^{n}$ be the Cartesian product. If $\mathcal{O}$ is the sheaf of germs of holomorphic functions on $\mathbf{C}^{n}$, then it is classical that $H^{q}(W, \mathcal{O})=(0)$ for all $q \geq 1$. In this paper, we study the cohomology on $\bar{W}$, the closure of $W$, of sheaves of germs of holomorphic functions on $W$ which satisfy certain boundary conditions on $\bar{W}$.

In this vein, several authors have studied particular examples of boundary behavior on product domains; e.g., differentiable boundary values by Bungart [2], Hölder continuous boundary values by Williams [6], and bounded boundary behavior by Stout [4], and by Andreotti and Stoll [1]. In this paper, we give a unified approach to many types of boundary behavior on product domains. In particular, we show that any class of functions which is closed under the integral operators

$$
T_{j} f=(2 \pi i)^{-1} \iint f\left(z_{1}, \cdots, \zeta, \cdots, z_{n}\right)\left(\zeta-z_{j}\right)^{-1} d \zeta \wedge d \bar{\zeta}
$$

defines a boundary behavior for which one obtains vanishing of cohomology. Moreover, using ideas of Douady [3], we explicitly construct fine resolutions for the sheaves of holomorphic functions that we consider. In this way, for example, we obtain vanishing of cohomology for sheaves of holomorphic functions with boundary behavior which is continuous; or Hölder continuous; or $m$-times continu-

Received by the editors Nove mber 8, 1971.

AMS 1970 subject classifications. Primary 32A30, 32C35; Secondary 32A30.

Key words and phrases. Sheaf cohomology, fine resolution, product domain, $L^{p}$ boundary behavior, continuous boundary behavior, differentiable boundary behavior with Hölder condition, Poincaré lemma, Dolbeault lemma.

(1) This paper incorporates part of the author's doctoral dissertation at Columbia University. 
ously differentiable, $1 \leq m \leq \infty$; or $L_{p}, 1 \leq p \leq \infty$.

Before introducing the full class of boundary behaviors we shall study, we first introduce some notation and some preliminary results. First we fix a base $\Omega$ for the relatively open sets in $\bar{W}$, consisting of relatively open products $U=U_{1} \times$ $U_{2} \times \cdots \times U_{n} \subset \bar{W}$. Thus each $U_{i} \subset \bar{W}_{i}$ is a relatively open plane set. We shall always assume that $\bar{W} \in \Omega$. For $U \in \Omega$, we let $C^{\infty}(\bar{U})$ denote the space of Whitney $C^{\infty}$-functions on $\bar{U}$, and we let $\mathscr{D}(U)$ denote the space of Schwartz distributions on $\stackrel{\circ}{U}$, the interior of $U$. For $f \in \mathcal{D}(U)$, we let $D_{j}(f)=\partial f / \partial \bar{z}_{j}$. Then $D_{j}$ is a linear map of $\mathcal{D}(U)$ into itself.

Next, let $F$ be a presheaf of abelian groups defined on $\Omega$ with restriction maps $r(U, V): F(U) \rightarrow F(V)$ if $V \subset U$, and $U, V \in \Omega$.

Definition 1. We say that $F$ is locally determined if the following two conditions are satisfied:

(a) If $V_{j} \in \Omega$ for $j \in J$, if $\bar{W}=\bigcup_{j \in J} V_{j}$, if $f \in F(\bar{W})$ and if $r\left(\bar{W}, V_{j}\right)(f)=0$ for all $j$, then $f=0$.

(b) If $V_{1}, \cdots, V_{m} \in \Omega$, if $\bar{W}=V_{1} \cup \cdots \cup V_{m}$, if $f_{j} \in F\left(V_{j}\right)$ and if $r\left(V_{j}, V_{j} \cap V_{k}\right)\left(f_{j}\right)=r\left(V_{\underline{k}}, V_{j} \cap V_{k}\right)\left(f_{k}\right)$ whenever the restriction maps are defined, then there exists $f \in F(\bar{W})$ so that $r\left(\bar{W}, V_{j}\right)(f)=f_{j}$ for all $j$.

Lemma 2. If $F$ is a locally determined presheaf on $\Omega$, and if $\mathcal{F}$ is the associated sheaf on $\bar{W}$, then $F(\bar{W}) \cong \Gamma(\bar{W}, \mathcal{F})$.

Proof. There is a canonical map $F(\bar{W}) \rightarrow \Gamma(\bar{W}, \mathcal{F})$. Condition (a) says that this map is injective. Since $\bar{W}$ is compact, condition (b) says that it is surjective.

We can now make precise our notion of boundary behavior.

Definition 3. Let $B$ be a locally determined presheaf of complex vector spaces on $\Omega$ satisfying

(a) For every $U \in \Omega, B(U)$ is a subspace of $\mathscr{D}(U)$, and restriction maps are just ordinary restrictions of distributions.

(b) For every $U \in \Omega$, if $f \in B(U), \phi \in C^{\infty}(\bar{U})$, then $(\phi f) \in B(U)$.

(c) For every $U \in \Omega$, for $f \in B(U)$, and for each $j$, there exists $g \in B(U)$ so that $D_{j}(g)=f$. Moreover, if $1 \leq j_{1}<\ldots<j_{q} \leq n$, if $j \notin\left\{j_{1}, \cdots, j_{q}\right\}$ and if $D_{j_{1}} \ldots D_{j_{q}}(f) \in B(U)$, then $D_{j_{1}} \ldots D_{j_{q}}(g) \in B(U)$. Finally, if $k \neq j$, and $D_{k} f=0$, then $D_{k} g \stackrel{q}{=} 0$.

For $U \in \Omega$, let $S(U)=\left\{f \in B(U) \mid D_{j} f=0\right.$ for all $\left.j\right\}$. Then $S$ is clearly a locally determined presheaf on $\Omega$ and we let $\mathcal{S}$ be the associated sheaf on $\bar{W}$.

Note that, formally, $B(U)$ and $S(U)$ are spaces of distributions on $\stackrel{\circ}{U}$. In the usual way we identify a locally integrable function $f \in L_{1}^{\text {loc }}(U)$ with the distribution:

$$
f(\phi)=\int_{U} f \phi d m \text { for } \phi \in C_{0}^{\infty}(U)
$$


where $d m$ is Lebesgue measures on $\mathbf{C}^{n}$. The following well-known regularity theorem allows us to identify elements of $S(U)$ with holomorphic functions on $\stackrel{O}{U}$. We omit the proof.

Theorem 4. Let $U \subset \mathrm{C}^{n}$ be open, and let $f \in \mathcal{D}(U)$. Suppose $D_{j} f=0$ for $j=1,2, \ldots, n$. Then $f$ is given in the sense of (1) above by a unique holomorphic function, which we also write as $f$.

For obvious reasons, we call $\mathcal{S}$ the sheaf of germs of holomorphic functions on $W$ with boundary behavior $B$. Our main result is that all the higher cohomology groups of $\mathcal{S}$ on $\bar{W}$ vanish (see Theorem 13). We shall also see that there are many examples of presheaves $B$ which satisfy Definition 3. Of the three conditions in Definition 3, the most stringent is clearly (c). This will be precisely the condition which allows us to prove a generalized Poincaré lemma (see Lemma 9). When $B(U)$ is a space of integrable functions on $\bar{U}$, condition (c) is implied by the statement that $B(U)$ is closed under certain integral operators (see the remark after Lemma 15). For the examples of boundary behavior we shall consider, this is then well known.

Before turning to examples, however, we proceed with the abstract approach, and first construct a fine resolution for the sheaf $\mathcal{S}$. Using ideas in Douady [3], we introduce

Definition 5. Let $U \in \Omega$.

(i) For each integer $p, 0 \leq p \leq n$, and for each sequence $0<i_{1}<i_{2}<\ldots<$ $i_{p} \leq n$, set $Q_{i_{1}}, \cdots, i_{p}(U)=\left\{f \in B(U) \mid\right.$ for any sequence $0<j_{1}<j_{2}<\ldots<j_{q} \leq n$ with $\left.\left\{i_{1}, i_{2}, \cdots, i_{p}\right\}^{p} \cap\left\{j_{1}, j_{2}, \cdots, j_{q}\right\}=\varnothing, D_{j_{1}} D_{j_{2}} \cdots D_{j_{q}}(f) \in B(U)\right\}$.

(ii) For each integer $p, 0 \leq p \leq n, E^{p}(U)$ is the vector space of differential forms of bidegree $(0, p)$ on $U$ such that the coefficient of $d \bar{z}_{i_{1}} \wedge d \bar{z}_{i_{2}} \wedge \ldots \wedge$ $d \bar{z}_{i_{p}}$ is an element of $Q_{i_{1}}, \cdots, i_{p}(U)$.

The rules which associate to each $U \in \Omega$ the spaces $Q_{i_{1}, \cdots, i}(U)$ and $E^{p}(U)$, together with the natural restriction maps, form locally determined presheaves on $\Omega$. Let $\mathcal{Q}_{i_{1}, \cdots, i_{p}}$ and $E^{p}$ be the associated sheaves on $\bar{W}$ so that

$$
\Gamma\left(\bar{W}, \mathcal{Q}_{i_{1}}, \cdots, i_{p}\right) \cong Q_{i_{1}, \cdots, i_{p}}(\bar{W}) \text { and } \Gamma\left(\bar{W}, \mathcal{G}^{p}\right) \cong E^{p}(\bar{W}) .
$$

Lemma 6. Let $U \in \Omega$, let $f \in Q_{i_{1}}, \cdots, i_{p}(U)$ and suppose $0<j_{1}<j_{2}<\ldots<$ $j_{q} \leq n$ with $\left\{i_{1}, \cdots, i_{p}\right\} \cap\left\{j_{1}, \cdots, j_{q}\right\}=\varnothing$. Then $D_{j_{1}} D_{j_{2}} \ldots D_{j_{q}}(f) \epsilon$ $Q_{\left(i_{1}, \cdots, i_{p}, j_{1}, \cdots, j_{q}\right)}(U)$ where $\left\langle i_{1}, \cdots, i_{p}, j_{1}, \cdots, j_{q}\right\rangle$ denotes the increasing re arrangement of these indicies.

Proof. It clearly suffices to prove this for the case $q=1$. Thus if $f \epsilon$ $Q_{i_{1}}, \cdots, i_{p}(U), j \notin\left\{i_{1}, \cdots, i_{p}\right\}$, then $D_{j}(f) \in B(U)$ by Definition $5(i)$. To show 
$D_{j} f \in Q_{\left\langle i_{1}, \cdots, i_{p}, j\right\rangle}(U)$ choose a sequence $0<k_{1}<\cdots<k_{r} \leq n$ with $\left\{i_{1}, \cdots, i_{p}, j\right\}$ $\cap\left\{k_{1}, \cdots, k_{r}\right\}=\varnothing$. Then $\left(D_{k_{1}} \cdots D_{k_{r}}\right)\left(D_{j} f\right)=\left(D_{k_{1}} \cdots D_{k_{r}} D_{j}\right)(f) \in B(U)$ since $\left\{i_{1}, \cdots, i_{p}\right\} \cap\left\{j, k_{1}, \cdots, k_{r}\right\}=\varnothing$.

We now define maps $\bar{\partial}_{U}: E^{p}(U) \rightarrow E^{p+1}(U)$ by setting

$$
\begin{aligned}
& \bar{\partial}_{U}\left(\sum_{i_{1}<\cdots<i_{p}} f_{i_{1}, \cdots, i_{p}} d \bar{z}_{i_{1}} \wedge \ldots \wedge d \bar{z}_{i_{p}}\right) \\
& \quad=\sum_{i_{1}<\ldots<i_{p}} \sum_{k=0}^{n} g_{k} d \bar{z}_{k} \wedge d \bar{z}_{i_{1}} \wedge \cdots \wedge d \bar{z}_{i_{p}}
\end{aligned}
$$

where $g_{k}=0$ if $k \in\left\{i_{1}, \cdots, i_{p}\right\}$ and $g_{k}=D_{k} f_{i_{1}}, \cdots, i_{p}$ if $k \notin\left\{i_{1}, \cdots, i_{p}\right\}$. Note that by Lemma $6, g_{k} \in Q_{\left\langle i_{1} \cdots, i_{p}, k\right\rangle}(U)$ so that $\bar{\partial}_{U}$ is well defined. The maps $\bar{\partial}_{U}$ clearly commute with restriction maps, and hence induce sheaf maps $\bar{\partial}: \mathcal{E}^{p} \rightarrow$ $\mathcal{G}^{p+1}$. For every $U \in \Omega$ we also have a natural inclusion map $S(U) \rightarrow E^{0}(U)$. This induces an inclusion of sheaves $\mathcal{S} \rightarrow^{i} \mathcal{E}^{0}$.

We thus get a long sequence of sheaf maps:

$$
(0) \rightarrow \mathcal{S} \stackrel{i}{\rightarrow} \xi^{0} \stackrel{\bar{\partial}}{\rightarrow} \xi^{1} \stackrel{\bar{\partial}}{\rightarrow} \ldots \stackrel{\bar{\partial}}{\rightarrow} \xi^{n-1} \stackrel{\bar{\partial}}{\rightarrow} \xi^{n} \rightarrow(0) .
$$

Since one can reverse order of differentiation for distributions the standard argument shows that sequence (2) is in fact a complex: $\bar{\partial} \circ i=0, \bar{\partial} \circ \bar{\partial}=0$. Our object is to show that the sheaves $\mathcal{E}^{p}$ are all fine, and that sequence (2) is exact.

The proof that $\mathcal{E}^{p}$ is fine requires a preliminary result.

Lemma 7. Let $U \in \Omega$, and let $0<j_{1}<\cdots<j_{q} \leq n$. For any sequence $0<$ $i_{1}<\cdots<i_{p} \leq n$ with $\left\{i_{1}, \cdots, i_{p}\right\} \cap\left\{j_{1}, \cdots, j_{q}\right\}=\varnothing$, if $f \in Q_{i_{1}, \cdots, i_{p}}(U)$ and if $\phi \in C^{\infty}(\bar{U})$, then $D_{j_{1}} D_{j_{2}} \cdots D_{j_{q}}(\phi f) \in B(U)$. In particular, it follows that $\phi f \in Q_{i_{1}, \cdots, i_{p}}(U)$.

Proof. The proof is by induction on $q$. The case $q=0$ follows from Definition $3(\mathrm{~b})$. For the induction step, assume that the lemma is true for any sequence $0<j_{1}^{\prime}<\cdots<j_{q-1}^{\prime} \leq n$. From the rule for differentiation of products,

$$
D_{j_{1}} \ldots D_{j_{q}}(\phi f)=\left(D_{j_{1}} \ldots D_{j_{q-1}}\right)\left(\phi D_{j_{q}} f\right)+\left(D_{j_{1}} \ldots D_{j_{q-1}}\right)\left(\left(D_{j_{q}} \phi\right) f\right) .
$$

Since $f \in Q_{i_{1}, \cdots, i_{p}}(U), D_{j_{q}} f \in Q_{\left\langle i_{1}, \cdots, i_{p}, j_{q}\right\rangle}(U)$ and applying the induction hypothesis to the sequence $0<j_{1}<\cdots<j_{q-1}$, we conclude that $\left(D_{j_{1}} \cdots D_{j_{q-1}}\right)\left(\phi D_{j_{q}} f\right) \in B(U)$. Similarly, since $\left(D_{j_{q}} \phi\right) \in C^{\infty}(\bar{U})$, $\left(D_{j_{1}} \cdots D_{j_{q-1}}^{q-1}\right)\left(\left(D_{j_{q}} \phi\right) f\right) \in B(U)$. Hence $\left(D_{j_{1}} \cdots D_{j_{q}}\right)(\phi f) \in B(U)$.

Proposition 8. The sheaves $\mathcal{E}^{p}, 0 \leq p \leq n$, are fine.

Proof. $\mathcal{E}^{p}$ is isomorphic to the direct sum of the sheaves $\mathscr{Q}_{i_{1}, \cdots, i_{p}}$ and thus 
it suffices to show that each $\mathcal{Q}_{i_{1}, \cdots, i_{p}}$ is fine. Let $\left\{V_{\alpha}\right\}, \alpha \in A$, be a locally finite open cover of $\bar{W}$. Let $\left\{\phi_{\alpha}^{i}\right\},{ }^{2}, \stackrel{i}{\in} A$, be a $C^{\infty}$-partition of unity subordinate to $\left\{V_{\alpha}\right\}$. For any $U \in \Omega$, define $\eta_{\alpha}: Q_{i_{1}}, \cdots, i_{p}(U) \rightarrow Q_{i_{1}, \cdots, i_{p}}(U)$ by $\eta_{\alpha}(f)=\phi_{\alpha} f$. By Lemma 7 , this is well defined, and the maps $\eta_{\alpha}$ commute with restriction maps. Hence we get a sheaf partition of unity subordinate to the open cover $\left\{V_{\alpha}\right\}, \alpha \in A$, so that the sheaf $\mathscr{O}_{i_{1}}, \cdots, i_{p}$ is fine.

Our next object is to show that the sheaf sequence (2) is exact. In fact, at the same time we shall show that the sequence of global sections is exact.

Lemma 9 (Ge neralized Poincaré Lemma). Let $U \in \Omega$, and let $f \in Q_{i_{1}, \cdots, i_{p}}(U)$. Suppose $D_{j}(f)=0$ for $j=i_{p}+1, \cdots, n$. Then there exists $g \in Q_{i_{1}}, \cdots, i_{p-1}(U)$ so that $D_{i_{p}}(g)=f$ and $D_{j}(g)=0$ for $j=i_{p}+1, \cdots, n$.

Proof. We let $g \in B(U)$ be the solution to $D_{i_{p}}(g)=f$ guaranteed by Definition $3(\mathrm{c})$. From that definition, since $D_{j}(f)=0$ for $j=i_{p}+1, \cdots, n$, we also have $D_{j}(g)=0$ for $j=i_{p}+1, \cdots, n$. Hence we only need to show that $g \in$ $Q_{i_{1}}, \cdots, i_{p-1}(U)$.

Choose a sequence $0<j_{1}<\cdots<j_{q} \leq n$ with $\left\{i_{1}, \cdots, i_{p-1}\right\} \cap\left\{j_{1}, \cdots, j_{q}\right\}$ $=\varnothing$. There are two possibilities. If $i_{p} \in\left\{j_{1}, \cdots, j_{q}\right\}$, say $i_{p}=j_{r}$, then

$$
\begin{aligned}
\left(D_{j_{1}} \ldots D_{j_{r}} \ldots D_{j_{q}}\right)(g) & =\left(D_{j_{1}} \ldots \hat{D}_{j_{r}} \ldots D_{j_{q}}\right)\left(D_{i_{p}} g\right) \\
& =\left(D_{j_{1}} \ldots \hat{D}_{j_{r}} \ldots D_{j_{q}}\right)(f) \in B(U)
\end{aligned}
$$

since $f \in Q_{i_{1}, \cdots, i_{p}}(U)$ and $\left\{j_{1}, \cdots, \hat{j}_{r}, \cdots, j_{q}\right\} \cap\left\{i_{1}, \cdots, i_{p}\right\}=\varnothing$. (Here ^ indicates omission of the operator or index.)

If $i_{p} \notin\left\{j_{1}, \cdots, j_{q}\right\}$, then $D_{j_{1}} \cdots D_{j_{q}}(f) \in B(U)$ since $f \in Q_{i_{1}, \cdots, i_{p}}(U)$. Again by Definition $3(\mathrm{c})$ we conclude that $D_{j_{1}} \cdots D_{j_{q}}(g) \in B(U)$. Hence $g \in$ $Q_{i_{1}, \cdots, i_{p-1}}(U)$.

Lemma 10 (Generalized Dolbeault Lemma). Let $U \in \Omega$ and let $\omega \in E^{p}(U)$. If $\bar{\partial}_{U} \omega=0$, there exists $\eta \in E^{p-1}(U)$ so that $\bar{\partial}_{U} \eta=\omega$.

Proof. We imitate the standard proof, and proceed by induction on $k$, the least integer such that $\omega$ involves only the differentials $d \bar{z}_{1}, \cdots, d \bar{z}_{k}$. If $k=$ $0, \omega=0$, and we may take $\eta=0$. In general, write

$$
\omega=\sum_{1 \leq i_{1}<\cdots<i_{p} \leq k} \omega_{i_{1}, \cdots, i_{p}} d \bar{z}_{i_{1}} \wedge \ldots \wedge d \bar{z}_{i_{p}} .
$$

Then we have

$$
\begin{aligned}
0 & =\bar{\partial}_{U} \omega \\
& =\sum_{1 \leq i_{1}<\ldots<i_{p} \leq k} \sum_{j \notin\left\{i_{1}, \cdots, i_{p}\right\}} D_{j}\left(\omega_{i_{1}}, \cdots, i_{p}\right) d \bar{z}_{j} \wedge d \bar{z}_{i_{1}} \wedge \ldots \wedge d \bar{z}_{i_{p}} .
\end{aligned}
$$


A comparison of coefficients shows that $D_{j}\left(\omega_{i_{1}}, \cdots, i_{p}\right)=0$ for $j=i_{p}+1, \cdots, n$. For each $\omega_{i_{1}, \cdots, i_{p}}$ with $i_{p}=k, \omega_{i_{1}, \cdots, i_{p-1}, k} \in Q_{i_{1}, \cdots, i_{p-1}}(U)$ and by Lemma 9 there exists $\gamma_{i_{1}, \cdots, i_{p-1}} \in Q_{i_{1}, \cdots, i_{p-1}}(U)$ so that

$$
D_{i_{p}} \gamma_{i_{1}}, \cdots, i_{p-1}=(-1)^{p-1} \omega_{i_{1}}, \cdots, i_{p} \text { and } D_{j} \gamma_{i_{1}, \cdots, i_{p-1}}=0
$$

for $j=i_{p}+1, \cdots, n$. Let $\gamma \in E^{p-1}(U)$ be the element

$$
\gamma=\sum_{i_{1}<\cdots<i_{p-1}} \gamma_{i_{1}, \cdots, i_{p-1}} d \bar{z}_{i_{1}} \wedge \cdots \wedge d \bar{z}_{i_{p-1}}
$$

By construction,

$$
\bar{\partial}_{U} \gamma=\sum_{i_{1}<\ldots<i_{p-1}<k} \omega_{i_{1}, \cdots, i_{p-1}, k} d \bar{z}_{i_{1}} \wedge \ldots \wedge d \bar{z}_{i_{p-1}} \wedge d \bar{z}_{k}+\delta
$$

where $\delta \in E^{p}(U)$ involves only the differentials $d \bar{z}_{1}, \cdots, d \bar{z}_{k-1}$. Let

$$
\beta=\sum_{1 \leq i_{1}<\cdots<i_{p}<k} \omega_{i_{1}}, \cdots, i_{p} d \bar{z}_{i_{1}} \wedge \cdots \wedge d \bar{z}_{i_{p}} .
$$

Then if we set $\phi=\omega-\bar{\partial}_{U} \gamma=\underline{\beta}-\delta \in E^{p}(U), \phi$ involves only the differentials $d \bar{z}_{1}, \cdots, d \bar{z}_{k-1}$, and $\bar{\partial}_{U} \phi=\bar{\partial}_{U} \omega-\bar{\partial}_{U} \bar{\partial}_{U} \gamma=0$.

Applying the induction hypothesis, there is $\psi \in E^{p-1}(U)$ with $\bar{\partial}_{U} \psi=\phi$. Then $\eta=\gamma+\psi \in E^{p-1}(U)$, and $\bar{\partial}_{U} \eta=\bar{\partial}_{U} \gamma+\bar{\partial}_{U} \psi=\bar{\partial}_{U} \gamma+\omega-\bar{\partial}_{U} \gamma=\omega$. This completes the proof.

Theorem 11. The sheaf sequence (2) is exact.

Proof. Let $z \in \bar{W}$. By Definition 3, the sequence $(0) \rightarrow \delta_{z} \rightarrow \mathcal{E}_{z}^{0} \rightarrow \mathcal{E}_{z}^{1}$ is exact. If $[\omega] \in \mathcal{G}_{z}^{p}$ with $\bar{\partial}[\omega]=0$, we can find $U \in \Omega$, and a representative $\omega \epsilon$ $E^{p}(U)$ for $[\omega]$ so that $\bar{\partial}_{U} \omega=0$. By Lemma 10 , there is $\eta \in E^{p-1}(U)$ so that $\bar{\partial}_{U} \eta=\omega$. But then $[\eta] \epsilon \mathcal{E}_{z}^{p-1}$ and $\bar{\partial}[\eta]=[\omega]$. Thus $\underset{z}{\mathcal{E}^{p-1}} \rightarrow \underset{z}{\mathcal{E}^{p}} \rightarrow \underset{z}{\mathcal{E}_{p}^{p+1}}$ is exact.

The ore $\mathrm{m}$ 12. The sequence of global sections

$$
(0) \rightarrow \Gamma(\bar{W}, \delta) \rightarrow \Gamma\left(\bar{W}, \mathcal{E}^{0}\right) \rightarrow \cdots \rightarrow \Gamma\left(\bar{W}, \mathcal{E}^{n}\right) \rightarrow(0)
$$

induced by sequence (2) is exact.

Proof. Since all the presheaves we are dealing with are locally determined, Lemma 2 shows that the sequence of global sections is just the sequence $(0) \rightarrow$ $s(\bar{W}) \rightarrow E^{0}(\bar{W}) \rightarrow \cdots \rightarrow E^{n}(\bar{W}) \rightarrow(0)$. Since $\bar{W} \in \Omega$, Lemma 10 again shows that if $\omega \in E^{p}(\bar{W})$ with $\bar{\partial}_{\bar{W}} \omega=0$, then there exists $\eta \in E^{p-1}(\bar{W})$ with $\bar{\partial}_{\bar{W}} \eta=\omega$. Thus the sequence

$$
E^{p-1}(\bar{W}) \rightarrow E^{p}(\bar{W}) \rightarrow E^{p+1}(\bar{W})
$$

is exact. Finally, exactness at $E^{0}(\bar{W})$ again follows from Definition 3. This completes the proof.

We now combine the last results to give our main theorem on the Čech Cohomology of the sheaf $\mathcal{S}$ on $\bar{W}$. 
Theorem 13. $H^{q}(\bar{W}, \mathcal{S})=(0)$ for all $q \geq 1$.

Proof. Sequence (2) gives a fine resolution for the sheaf $\mathcal{S}$ by Proposition 8 and Theorem 11. Hence

$$
H^{q}(\bar{W}, \mathcal{S}) \cong \frac{\left\{\omega \in \Gamma\left(\bar{W}, \mathcal{E}^{p}\right) \mid \bar{\partial} \omega=0\right\}}{\left\{\bar{\partial} \eta \mid \eta \in \Gamma\left(\bar{W}, \mathcal{E}^{p-1}\right)\right\}} .
$$

By Theorem 12, this last quotient is zero, which completes the proof.

We now give some applications of Theorem 13 by exhibiting presheaves $B$ satisfying Definition 3. In these examples, $B(U)$ will always be some subspace of integrable functions on $\bar{U}$; i.e. $B(U) \subset L_{1}(\bar{U})$. For such functions, we can define a linear right inverse $T_{j}$ to the linear mapping $D_{j}$.

Lemma 14. Let $U=U_{1} \times \cdots \times U_{n} \subset \subset \mathbf{C}^{n}$, and let $f \in L_{1}(\bar{U})$. Then as a function of $z=\left(z_{1}, \cdots, z_{n}\right) \in \bar{U}$,

$$
T_{j} f(z)=\frac{1}{2 \pi i} \int_{U_{j}} \frac{f\left(z_{1}, \cdots, \zeta, \cdots, z_{n}\right)}{\zeta-z_{j}} d \zeta \wedge d \bar{\zeta}
$$

exists almost everywhere, and $T_{j} f \in L_{1}(\bar{U})$.

Proof. This is an obvious modification of the corresponding one-dimensional result. See Vekua [5, Theorem 1.13, p. 28].

Lemma 15. Let $U=U_{1} \times \cdots \times U_{n} \subset \subset \mathbf{C}^{n}$, and let $f \in L_{1}(\bar{U})$. Then

(i) $D_{j} T_{j}(f)=f$ for $j=1,2, \cdots, n$. $L_{1}(\bar{U})$, then

(ii) If $1 \leq j_{1}<\cdots<j_{q} \leq n$, if $j \notin\left\{j_{1}, \cdots, j_{q}\right\}$ and if $\left(D_{j_{1}} \cdots D_{j_{q}}\right)(f) \epsilon$

$$
\left(D_{j_{1}} \ldots D_{j_{q}}\right)\left(T_{j} f\right)=T_{j}\left(D_{j_{1}} \ldots D_{j_{q}}(f)\right) .
$$

Again, the proof, which we omit, is standard. Now suppose $B(U) \subset L_{1}(\bar{U})$, and suppose $T_{j}: B(U) \rightarrow B(U)$ for every $j$. Then clearly $B(U)$ satisfies condition (c) in Definition 3, for we simply take $g=T_{j} f$. Thus in examples, it suffices to show that $B(U)$ is closed under the operators $T_{j}$ for every $U \in \Omega$ and for every $j$.

Example 1. Let $W_{1}{ }^{\circ} \subset \subset C, i=1,2, \cdots, n$, be relatively compact domains with boundary of plane measure zero. Let $W=W_{1} \times \cdots \times W_{n} \subset \subset \mathbf{C}^{n}$, and let $\Omega$ be the base of relatively open sets of $\bar{W}$ consisting of all relatively open products with boundary of measure zero. Choose a number $p, 1 \leq p \leq \infty$, and for $U \in \Omega$, let $B_{p}(U)=L_{p}(U)$. If $\bar{W}=\bigcup_{i=1}^{m} U_{i}$ and if $f / U_{i} \in L_{p}\left(U_{i}\right)$ for $i=$ $1, \cdots, m$, then certainly $f \in L_{p}(\bar{W})$. Hence $B_{p}$ is a locally determined presheaf on $\Omega$ and Definition $3(a)$ is satisfied. Since $L_{p}(U)$ is closed under multiplication by elements of $C^{\infty}(\bar{U})$, Definition $3(\mathrm{~b})$ is satisfied. Definition $3(\mathrm{c})$ is a consequence of the following: 
Lemma 16. Let $U=U_{1} \times \cdots \times U_{n} \subset \subset \mathbf{C}^{n}$, and let $f \in L_{p}(\bar{U})$. Then $T_{j} f \in$ $L_{p}(\bar{U})$ for $j=1,2, \cdots, n$.

Proof. Again this is an obvious modification of the one-dimensional result. See Vekua [5, Theorem 1.13, p. 28, Theorem 1.19, p. 38].

Let $S_{p}$ and $S_{p}$ be the presheaf and sheaf of holomorphic functions with boundary behavior $B_{p}$. Note that again, for $z \in W,\left(\mathcal{S}_{p}\right)_{z}=\mathcal{O}_{z}$, but for $U \in \Omega, \Gamma\left(U, \mathcal{S}_{p}\right)$ $=\Gamma(\stackrel{\circ}{U}, \Theta) \cap L_{p}^{\operatorname{loc}}(U)$. As a consequence of Theorem 13, we obtain

Theorem 17. $H^{q}\left(\bar{W}, \delta_{p}\right)=(0)$ for all $q \geq 1$.

Example 2. We first introduce some notation. Let $\Gamma$ be a simple rectifiable Jordan plane curve, so that its equation may be written $z(s)=x(s)+i y(s)$ where $s$ is arc length from some fixed point. We say that $\Gamma$ is of class $C(m, \alpha), 0 \leq m \leq$ $\infty, 0 \leq \alpha \leq 1$, if all derivatives of $z$ of order $\leq m$ are continuous, and if every $m$ th derivative satisfies a Hölder condition of order $\alpha$. If $D \subset C$ is a plane domain, we say that $D$ is of class $C(m, a)$ if the boundary of $D$ consists of a finite number of simple rectifiable Jordan curves of class $C(m, \alpha)$. If $f$ is a complex-valued function on $D \subset C^{n}$, we say that $f$ is of class $C(m, \alpha)$ if every derivative of order $\leq m$ is continuous on $\bar{D}$ and if every derivative of order $m$ satisfies a Hölder condition of order $\alpha$.

Now fix $m, \alpha, 0 \leq m \leq \infty, 0 \leq \alpha \leq 1$. Let $W_{i} \subset \subset \mathbf{C}$ for $i=1,2, \cdots, n$ be relatively compact domains with each $W_{i}$ of class $C(m+1, \alpha)$. Let $W=W_{1} \times \cdots$ $\times W_{n}$. Let $\Omega$ be the set of all relatively open products $U=U_{1} \times \cdots \times U_{n} \subset \bar{W}$ such that each $U_{i}$ is of class $C(m+1, a)$. (These clearly form a base for the relatively open subsets of $\bar{W}$.) For $U \in \Omega$, let $B(m, \alpha)(U)=C(m, \alpha)(\stackrel{O}{)}$, the space of complex functions of class $C(m, \alpha)$ on $\stackrel{\circ}{U} B(m, \alpha)$ is a locally determined presheaf on $\Omega$, and $B(m, \alpha)(U)$ is closed under multiplication by elements of $C^{\infty}(\bar{U})$. Hence Definition $3(\mathrm{a})$ and (b) are satisfied. Definition $3(\mathrm{c})$ is a consequence of

Lemma 18. Let $U=U_{1} \times \cdots \times U_{n}$ where each $U_{j}$ is of class $C(m+1, \alpha)$. Then $T_{j} f \in C(m, \alpha)(U)$ if $f \in C(m, \alpha)(U)$ for $j=1,2, \cdots, n$.

Proof. A stronger result is actually true since the operator $T_{j}$ increases the differentiability with respect to the $j$ th variable. The proof is a modification of the one-dimensional result. See Vekua [5, Theorem 1.32, p. 56].

Let $S(m, \alpha), \mathcal{S}(m, \alpha)$ be the presheaf and sheaf of holomorphic functions with boundary behavior $B(m, a)$. It is clear that if $z \in W, S(m, a)_{z}=\mathcal{O}_{\boldsymbol{z}}$, while if $z \in \bar{W}-W, \mathcal{S}(m, a)_{z}$ is the ring of germs of functions at $z$ which are of class $C(m, \alpha)$ on some neighborhood of $z$ in $\bar{W}$, and which are holomorphic on $W$. As a consequence of Theorem 13 we obtain 
Theorem 19. If $W=W_{1} \times \cdots \times W_{n}$, and if each $W_{j}$ is of class $C(m+1, a)$, then $H^{q}(\bar{W}, \mathcal{S}(m, \alpha))=(0)$ for all $q \geq 1$.

For the sheaves $\mathcal{S}(m, \alpha)$, there is a well-defined map $\mathcal{S}(m, \alpha)_{\boldsymbol{z}} \rightarrow \mathcal{S}(m, \alpha)_{\boldsymbol{z}}$ given by $f \rightarrow \exp (2 \pi i f)$. Moreover, if $f \in \mathcal{S}(m, \alpha)_{z}$ and if $f$ is invertible, then $(1 / 2 \pi i) \log (f) \in \mathcal{S}(m, \alpha) z_{z}$. If we let $\mathcal{S}(m, \alpha)^{*}$ denote the sheaf of invertible elements of $\mathcal{S}(m, a)$, we get the standard exact sequence:

$$
(0) \rightarrow \mathbf{Z} \rightarrow \mathcal{S}(m, a) \stackrel{\exp (2 \pi i)}{\longrightarrow} \mathcal{S}(m, \alpha)^{*} \rightarrow(0) .
$$

Applying Theorem 19 to the resulting long exact sequence of cohomology groups, we obtain

Theorem 20. $H^{q}\left(\bar{W}, S(m, \alpha)^{*}\right) \cong H^{q+1}(\bar{W}, \mathbf{Z})$. In particular, if eacb $W_{i}$ is simply connected, $H^{q}\left(\bar{W}, \mathcal{S}(m, \alpha)^{*}\right)=(0)$.

Example 3. Taking $m=\alpha=0$ in Theorem 19, we see that if each $W_{j}$ is of class $C(1,0)$, then the sheaf of germs of continuous functions on $\bar{W}$ which are holomorphic on $W$ has zero higher cohomology. For this sheaf $\mathcal{S}(0,0)=\mathcal{S}$, the requirement of smoothness of boundary is not necessary since if $U=U_{1} \times \cdots \times$ $U_{n} \subset \subset \mathbf{C}^{n}$ is arbitrary, and if $f$ is continuous on $\bar{U}$, then $T_{j} f$ is also continuous. Thus we obtain

Theorem 21. For an arbitrary product domain $W \subset \subset \mathrm{C}^{n}$, if $\mathcal{S}$ is the sheaf of germs of continuous functions on $\bar{W}$ which are bolomorphic on $W$, then $H^{q}(\bar{W}, \mathcal{S})=(0)$ for all $q \geq 1$.

\section{REFERENCES}

1. A. Andreotti and W. Stoll, The extension of bounded holomorphic functions from hypersurfaces in a polycylinder, Proc. Conf. in Complex Analysis, 1969, Rice University Studies, Spring 1970.

2. L. Bungart, Cartan's Theorem B for domains with boundary, Notices Amer. Math. Soc. 16 (1969), 647-648. Abstract \#666-32.

3. A. Douady, Le problème des modules pour les sous-espaces analytiques compacts d'un espace analytique donné, Ann. Inst. Fourier (Grenoble) 16 (1966), fasc. 1, 1-95. MR $34 \# 2940$.

4. E. L. Stout, The second Cousin problem with bounded data, Pacific J. Math. 26 (1968), 379-387. MR 38 \#3467.

5. I. N. Vekua, Generalized analytic functions, Fizmatgiz, Moscow, 1959; English transl., Addison-Wesley, Reading, Mass., 1962. MR $21 \# 7288 ;$ MR $27 \# 321$.

6. E. R. Williams, The Poincaré lemma with estimates, Thesis, Columbia University, New York, 1970. 